\title{
Acción técnica, interacción social y práctica cotidiana: propuesta interpretativa de la tecnología
}

\author{
Technical action, social interaction and everyday practice: proposed interpretation \\ of technology
}

\author{
Manuel Calvo Trias (*) \\ Jaime García Rosselló (*)
}

\section{RESUMEN}

En este artículo se defiende el potencial del análisis tecnológico concebido como práctica eminentemente social. Esta perspectiva combina la información derivada de los análisis de las acciones y gestos técnicos con otros conceptos como el savoir faire tecnológico del grupo, los procesos de aprendizaje, las prácticas tecnológicas socialmente constituidas, etc. Esta estrategia metodológica permite adentrarse en el conjunto de praxis sociales que se van generando en el día a día de la fabricación y uso de los objetos e intervienen, de manera significativa, en la construcción de la arena social de las comunidades. Como caso de estudio de esta propuesta se citan ejemplos de la producción de la cerámica a mano

\begin{abstract}
This article is focused on the potential of technological analysis of material culture understood as an eminently social practice. This perspective allows us to incorporate all the information derived from the analysis of technical gestures with other concepts such as the technological know-how of the group, learning processes, socially constructed technological practices, etc. Thus, these concepts permit to establish methodological strategies from technological analysis that go beyond pure technical gestures. Furthermore, this procedure allows us to go into the set of daily social praxis that are developed in the production and use of objects which are significantly involved in the construction of the social arena of the communities. As a case study of this proposal we cited diferents examples of the handmade production of pottery.
\end{abstract}

(*) Grupo Arqueobalear. Departamento de Ciencias Históricas y Teoría de las Artes, Universidad de Les Illes Balears. C/ Valldemosa km 7,5. Palma de Mallorca.

Correos e.: manuel.calvo@uib.es; jaume.garcia@uib.es

Recibido 15-I-2013; aceptado 21-III-2013.
Palabras clave: Antropología de las técnicas; Historiografía de la tecnología; Cadena operativa; Habitus; Conocimiento tecnológico; Transmisión y aprendizaje tecnológico; Producción cerámica a mano.

Key words: Anthropology of techniques; Historiography of technology; Operatorial chain; Habitus; Technological knowledge; Technological learning; Handmade pottery production.

\section{INTRODUCCIÓN}

La tecnología es, sin lugar a dudas, una compleja práctica social y marca significativamente, una forma muy humana de expresarse en el mundo. Por ello, el estudio de la tecnología ha tenido y tiene un lugar privilegiado en arqueología. Una amplia diversidad de discursos disciplinares han girado en torno al análisis de las técnicas e innovaciones tecnológicas a través del tiempo y del espacio. En este artículo reflexionamos sobre la complejidad de los procesos e interacciones que se vinculan con el concepto de tecnología y analizamos su potencial interpretativo.

Si bien la mayor parte de las referencias a estrategias tecnológicas utilizadas en el texto se relacionan con la fabricación, uso y abandono de objetos, en realidad, defendemos un concepto más amplio de tecnología que va más allá de la manera con la que los seres humanos interactúan con los objetos y se extiende al conjunto de estrategias que las comunidades desarrollan a la hora de interactuar, gestionar y modificar los recursos naturales. Sin embargo, entendemos que el proceso tecnológico no es únicamente la secuenciación de acciones físicas de modificación 
de la materia, sino que implica la presencia de complejos esquemas mentales y sociales que se han ido configurando a través del tiempo dando lugar a una determinada tradición tecnológica. En este sentido, materiales, técnicas y personas se organizan en códigos socialmente construidos por lo que es el espacio social con un marcado carácter contingente quien dota de verdadero sentido a las opciones tecnológicas que se desarrollan.

El discurso que proponemos en este trabajo se ha estructurado en cuatro grandes bloques. En los dos primeros se hace un breve repaso a las visiones y perspectivas de análisis arqueológico del hecho tecnológico: las aproximaciones insertas en el Evolucionismo Histórico, en el Historicismo Cultural, en las aportaciones procesuales y, finalmente, en los enfoques postprocesuales. El tercer bloque desarrolla los aspectos y conceptos que, a nuestro modo de ver, permiten construir el andamiaje teórico-conceptual sobre el que asentar una visión de la tecnología como un fenómeno eminentemente social. Esta visión debe parte de su desarrollo a dos líneas discursivas que entendemos convergentes: unas se derivan, desde el ámbito francófono, de la Antropología de las Técnicas y las otras del marco conceptual derivado de la Social Agency Theory. Acabamos este artículo proponiendo algunas de las estrategias metodológicas que permiten avanzar en el estudio de esta visión de la tecnología. Para ello utilizamos como referencia, los procesos de fabricación de la cerámica a mano en ámbitos domésticos.

\section{DE "LOS OBJETOS SIN MANOS A LAS MANOS SIN CUERPO". LA PERSPECTIVA TECNOLÓGICA HASTA LA LLEGADA DE LAS CORRIENTES POSTPROCESUALES}

La tecnología ha tenido un papel fundamental en el desarrollo de las teorías interpretativas en arqueología. Muchas de las primeras periodizaciones cronoculturales (Mortillet, Lubbock, Lartet, Boucher de Perthes, Thomsen, Worsae, Montelius...) han utilizado, como criterio diferenciador, la variable tecnológica en su vertiente tipológica que también posibilitó la seriación y definición de las culturas (Childe 1936, 1956, 1988; Daniel 1987; Trigger 1992). El uso de los conceptos de fósil director y cultura material permitió clasificar, principalmente, los objetos y aso- ciarlos a un complejo cultural determinado. Este se extendió y asimiló después a pueblos, grupos o etnias prehistóricas. En la mayoría de casos, el análisis profundo de los aspectos formales y tipológicos de los objetos, no consideró ni a fabricantes, ni a usuarios. Se olvidaron "las manos" que los había creado y el espacio social donde fueron fabricados y usados. Este enfoque tecnológico también correlacionaba el desarrollo tecnológico de una comunidad y su desarrollo social (Childe 1988; Daniel 1963, 1967; Sabloff y Willey 1967; Trigger 1989, 1992), reforzando y justificando un discurso que reflejaba el predominio de la "Europa-metrópolis" sobre los territorios que, en ese momento, se estaban colonizando (Hernando 1992). El marco ideológico que inspiraba estas visiones se reflejó, como comentan autores como Fabian (Dobres 2000: 64), en el depósito y exhibición de los "objetos primitivos de los nativos" en los museos de ciencias naturales, y el de los colonizadores europeos en los museos de historia cultural.

En cierta manera, se estaba reflejando la idea central en el pensamiento occidental, de que la tecnología permitía "domesticar" la naturaleza. Se polarizaba así la división entre esta y la sociedad (Ingold 1990). Su influjo a la hora de asociar complejidad tecnológica y social fue determinante. Esta correlación potenció el uso del criterio tecnológico como un termómetro válido para el análisis de la complejidad social de los grupos humanos, asimilando la sencillez tecnológica a lo primitivo y la complejidad a lo civilizado.

El nacimiento de la Nueva Arqueología y su desarrollo procesual (Binford 1962, 1964, 1965; Fritz y Plog 1970; Watson et al. 1971; Clarke 1976, 1984) reacomoda los conceptos de cultura y tecnología. La cultura se concibe como un medio extrasomático de adaptación e interacción con el medio (Binford 1965). A su vez, mediante el análisis de la tecnología, se quiso establecer algún tipo de patrón transcultural que explicase las regularidades observadas en el registro arqueológico. El individuo pasó a considerarse un ente relativamente pasivo, reflejo del medio que lo rodeaba. Aumentó el interés por el estudio de las materias y técnicas asociadas a la fabricación de los objetos, pero sin profundizar en el carácter eminentemente social y activo de la tecnología. "Las manos que fabricaron el objeto" se tuvieron en cuenta, pero no el cuerpo social que estaba tras ellas, ni las consecuencias interpretativas que se 
derivaban de ello. Toda trasformación cultural se concibió como un resultado adaptativo externo y ambiental. Los componentes tecnológicos estaban fuertemente condicionados además por aspectos funcionales y, a través de ellos, se mejoraba la adaptación de las comunidades a su cambiante entorno. Ello condicionaba una determinada elección tecnológica que, a su vez, de manera bastante hierática, ordenaba las relaciones sociales de producción y uso de la tecnología (Dobres 2000).

En este planteamiento positivista subyacían visiones actualistas derivadas de los principios generales del Formalismo Económico. Los conceptos de energía, maximización de esfuerzos, efectividad tecnológica, áreas de captación de recursos, análisis de costes y beneficios, utilizados desde un prisma evolucionista adaptativo para normativizar comportamientos, se convirtieron en herramientas fundamentales en la interpretación. La separación de la tecnología del espacio social donde se generaba estaba en consonancia con dos tendencias claramente definidas en la sociedad moderna: la alienación de las personas respecto a su trabajo y la objetivación de la naturaleza como entidad controlable por la tecnología (Dobres y Hoffman 1994). Estas, a su vez, se relacionan con los postulados epistemológicos que beben del esquema de racionalidad derivado de un contexto cultural industrializado y capitalista. Dobres (2000) ve un ejemplo paradigmático de esta visión en la teoría defendida, entre otros, por Testart $(1982,1988)$ y analizada críticamente por Ingold (1988).

Dicha teoría, a través de un mecanismo unidireccional, explicaba la sencilla organización social de los cazadores-recolectores como resultado de una movilidad que les impedía un alto desarrollo tecnológico.

Una variante procesual más sistémica concibió la práctica tecnológica como un subsistema tecnoeconómico (Sabloff y Willey 1967; Thomas 1974; Clarke 1984) donde la cultura se entendía como parte del engranaje que daba cobertura a las necesidades de adaptación y a los demás condicionantes exógenos (Clarke 1984). Los modelos se basaban en el principio de que el análisis funcional de las sociedades requería su partición interna en subsistemas o esferas. Dicha conceptualización articulaba una dialéctica entre sociedad y tecnología, donde ambas eran partes del sistema mediante el cual el grupo se adaptaba al medio cambiante, sin que los agentes que inventaban, fabricaban y usaban la tecnología tuviesen un papel relevante en el proceso de innovación y cambio.

Otros autores entendían que los condicionantes sociales imponían dificultades que debían superarse para atender las exigencias tecnológicas (Testart 1982; Hayden 1995, 1998). En esta línea unidireccional de causa-efecto, el análisis tecnológico de los productos, de sus exigencias y de las respuestas adaptativas que generaban sería el camino para analizar la naturaleza de la organización de la producción y las exigencias sociales derivadas de ellas (Torrence 1983; Rice 1987; Roux y Matarasso 1999). El planteamiento se fundamentaba en la premisa de que "la necesidad es la madre de la invención", por lo que una variante surgiría de las demandas medioambientales y otra de las exigencias del propio desarrollo tecnológico (Pfafenberger 1988).

La tecnología, entendida como la respuesta más eficaz para gestionar los recursos naturales, estructuró estrategias de investigación que pretendían conocer el grado de control sobre la naturaleza de una cultura y sociedad a través de los objetos fabricados y del modo de hacerlos y usarlos. Para ello se optó por una analítica muy cuantitativa y tecnificada, en teoría, neutra y materialista, circunscrita casi en exclusiva a estudiar los procesos secuenciales que acababan configurando un objeto (Bettinger 1980; Torrence 1983). La tecnología se separaba de la sociedad, de las personas o del grupo en que se insertaba, así como del contexto cronocultural. La obtención de materias primas, la producción y diseño de los objetos, su funcionalidad y morfometría se veían como aspectos básicos y, en muchos casos, exclusivos del análisis tecnológico. Con el acercamiento positivista se incorporaban visiones actualistas formalistas (Leone 1982; Wallace 1986). Desde diferentes aproximaciones teóricas (Marcuse 1968; Habermas 1970; Mitcham 1994; Pippin 1995) se ha puesto de relieve la relación subyacente de los autores que gestaron y construyeron este edificio de base materialista, racionalista e instrumentalista con su entorno cultural. Otra implicación del enfoque fue la separación conceptual entre el agente creador de la tecnología (artesanos y artesanas, sus relaciones sociales, sus valores y creencias, el espacio social que ocupaban, etc.) y los objetos físicos que creaba (Dobres 2010). Esta ruptura se tradujo en una 
lectura incompleta del fenómeno al no concebir la tecnología como la compleja práctica social que es.

\section{LAS "MANOS CON CUERPO Y EL CUERPO CON SENTIMIENTO". LAS APORTACIONES POSTPROCESUALES}

En estas últimas décadas una revisión crítica del concepto de tecnología la ha redefinido como una herramienta básica en el estudio de las sociedades pretéritas, pretendiendo, en cierta manera, superar las visiones propias de la centralidad contemporánea de la tecnología. En esta revisión se ha enfatizado su carácter contingente (Leone 1982; Dobres 2010) (1) y se ha profundizado en el análisis de las relaciones estructuradas y estructurantes que se establecen entre objetos y sujetos. Ni los primeros ocupan una posición central, ni se crean divisiones artificiales entre objetos y personas, entre procesos tecnológicos, agentes y espacios sociales, entre género y trabajo, entre valores, relaciones de poder y proceso tecnológico. Todo ello se integra, a su vez, en el quehacer diario de la fabricación, uso, intercambio y abandono de los objetos, cuya praxis influye en una determinada manera de concebir, estructurar, reproducir o subvertir el mundo.

Ya el concepto de tecnología de Mauss en los 1930 establecía las bases para analizar este fenómeno como construcción social: technique as an action which is effective and tradicional (Mauss 1979: 104). Según Lemonnier (1992), como para Mauss la tecnología, en tanto que tradición, es transmitida y utilizada por las personas durante un largo espacio de tiempo e incorpora al concepto una clara carga social. La tecnología es usada, aprendida y mantenida como algo inherente a la propia sociedad que la ejecuta. El objeto no es un producto natural o fabricado que permite una mejor adaptación al medio sino que, en sí mismo, está conformado y definido por una realidad social particular (Mauss 1968). La tecnología, concebida como una práctica razonada, se desarrolla

(1) Is a continually unfolding process of social, meaningful, and sensuous engagement (a verb of action and interaction) engendered by social agents during their everyday activities of object making and use in historically and culturally circumscribed setting (Dobres 2000: 61). y trasmite en un contexto social donde el gesto técnico es interpretado como un acto consciente del individuo y de la colectividad social. Otro precursor de esta acepción de la tecnología sería Leroi-Gourham $(1964,1988,1989)$, tanto por su concepción de útil, como por la idea de que el comportamiento tecnológico humano es esencialmente colectivo, en cuanto constituido por la totalidad de las operaciones conocidas y estructuradas socialmente y utilizadas con un fin social. En esta línea de pensamiento son centrales los conceptos de aprendizaje y transmisión de conocimientos en tanto que fenómenos socialmente constituidos.

En estas últimas décadas muchas de las aportaciones básicas han surgido bien de las tendencias postprocesuales a través de la Social Agency Theory en el ámbito anglosajón, bien de la escuela de la Antropología de las Técnicas en el ámbito francófono. Como resultado, la tecnología ha adquirido una nueva dimensión. La cultura material es concebida como un agente activo, ya desde los primeros trabajos de Hodder $(1982,1989)$ o Tilley (1989). A su vez, entienden que la tecnología está íntimamente ligada a la sociedad o al grupo que la produce, así como a sus esquemas de racionalidad, en una relación estructurada y estructurante, donde el agente o persona que fabrica y usa el objeto en su quehacer cotidiano es el eje articulador del discurso tecnológico.

Por influencia del Estructuralismo y luego del Postestructuralismo inicialmente se prestó atención a los símbolos, las ideas y otros signos de representación mental articulados a través de una cultura material, cargada de un inevitable significado cultural. Se asignó un papel central al estudio de los aspectos cognitivos en la interpretación arqueológica. La estrategia de análisis utilizada inicialmente por Hodder, y seguida por otros (Tilley 1989, 1991a, 1991b; Shanks y Tilley 1987), fue concebir metafóricamente la cultura material como un texto. Sin embargo, esta estrategia semióticas fue criticada, con posterioridad, por quienes entendían que la cultura material era mucho más que un mero contenedor de aspectos ideológico-simbólicos (Lemonier 1986; Dietler y Herbich 1998; Olsen 2003, 2010).

Desde esas fechas de mediados de los 1980, la disciplina arqueológica ha ido incorporando conceptos y planteamientos, utilizados en especial desde la Sociología y la Filosofía, para analizar las interacciones entre las personas, los ob-

Trab. prehist., 71, N. ${ }^{\circ}$ 1, enero-junio 2014, pp. 7-22, ISSN: 0082-5638

doi: $10.3989 /$ tp. 2014.12121 
jetos y las estructuras en el seno de una sociedad (Ritzer y Gindoff 1994). Su finalidad era romper una cierta polarización existente entre las aproximaciones materialistas que contemplaban a la sociedad como una entidad más allá del individuo (Marxismo, Funcionalismo, Procesualismo, etc.) y otras más idealistas (Estructuralismo, Postestructuralismo), donde la explicación de los fenómenos sociales estaba más en los individuos y en sus esquemas mentales (Grau 2007). Aquí radican las propuestas que, desde la teoría social contemporánea, conciben la sociedad como estructura y agencia. Asumen que el individuo interactúa en el marco de una estructura social que lo condiciona pero, al conceptuarlo como un agente activo en su quehacer diario, contribuye a crear las condiciones que aseguran la reproducción o el cambio de dicha estructura. En este sentido, entre individuo, estructura y objeto (que tampoco se concibe como un elemento pasivo, sino como un agente activo), se producen una serie de interacciones de doble dirección que tienen unas características estructurantes y estructuradas sobre los objetos, los individuos y las mismas condiciones sociales.

\section{REPLANTEANDO LA TECNOLOGÍA COMO UN FENÓMENO EMINENTEMENTE SOCIAL}

Este trabajo parte de la premisa de que las interacciones que se dan en el espacio social entre las estructuras, los objetos y los individuos en el quehacer cotidiano condicionan e influyen en la reproducción o renegociación social. Consideramos central a ese respecto el concepto de habitus de Bourdieu $(1991,1997)$ y las aportaciones de la Teoría de la Estructuración de Giddens (1979, 1984). Sin embargo, en este trabajo no pretendemos analizar toda la riqueza conceptual que contienen, ni sus aplicaciones arqueológicas, si no enfatizar algunos aspectos pertinentes en nuestro discurso.

Giddens ha analizado en profundidad la estructura y la acción individual. El individuo, en tanto que agente activo en su quehacer cotidiano, tiende a entender, percibir y utilizar las reglas sociales y con ello, las reproduce, modifica y estructura de forma creativa. Es decir, puede reforzar o transformar las reglas de la estructura social a partir de la creatividad y la innovación.
Giddens define esta "conciencia práctica" como un conocimiento no discursivo de las instituciones sociales. Con ello, permite la acción del individuo en el seno de la estructura como un elemento sustentante o modificador de la misma, ya que: a) en ocasiones los individuos no son conscientes de ciertos aspectos de sus acciones; b) los agentes aplican a las situaciones un conocimiento incompleto de las normas sociales a partir de una gama diferente de estrategias; c) a menudo las acciones sociales no generan consecuencias directas. En la acción del agente actúan también: a) otros agentes, grupos, afiliaciones, o comunidades; b) el contexto espacial y el entorno mediambiental; c) los condicionantes sociales e históricos precedentes; d) las relaciones de poder establecidas en el espacio social. Esta línea de reflexión es esencial para entender el planteamiento tecnológico que se desprende de nuestra propuesta: excluir la consideración de las personas como autómatas que, inconscientemente, aplican las normas sociales.

Otra herramienta conceptual y metodológica utilizada es el concepto de habitus, definido como "estructura estructurante que organiza las prácticas y la percepción de las prácticas" (Bourdieu 1988: 170). Sería un esquema individual de disposiciones internas inconscientes que determinan cómo el individuo percibe y actúa en el mundo y que están estructuradas y estructuran el sistema externo. Mediante este concepto Bourdieu relaciona lo objetivo, la posición en la estructura social, y lo subjetivo, la interiorización de ese mundo objetivo. El habitus no sería una simple serie de normas sociales exteriores a los individuos, sino todo un conocimiento incorporado en cada uno de ellos, compuesto por estructuras que dan forma a las prácticas sociales y a la vez son estructuradas (adquieren forma mediante esas mismas prácticas y por lo tanto pueden ser manipuladas y cambiadas) (Bourdieu 1991: 89).

La aplicación del trabajo teórico de Bourdieu al proceso tecnológico ofrece un medio para situar a la cultura material y a las cadenas operativas en el esquema de interacción entre el agente y las estructuras. En el quehacer cotidiano de las personas que fabrican y usan los objetos se van generando rutinas tecnológicas que acaban condicionando su manera de ver y percibir el mundo. $\mathrm{Y}$ viceversa, esa visión del mundo y esos esquemas sociales estructuran a los agentes y condicionan la fabricación y uso diario de los objetos. Los 
procesos tecnológicos, cómo otros fenómenos de la actividad social, se formarían a través del $h a-$ bitus y de la interacción entre objeto, agente y estructura. La implicación es la incorporación de unos patrones de elección cultural a través de la práctica continuada. Las disposiciones, elecciones y percepciones de las posibilidades técnicas, insertas en el conjunto de relaciones sociales y configuradas mediante el habitus, llegarán a ser percibidas como naturales y perfectamente lógicas, más allá de consideraciones de eficiencia o eficacia de técnicas y materiales.

La práctica suele verse como predeterminada y estática. En cambio, el concepto bourdiano de habitus la configura como un fenómeno relacional dinámico. Es un producto a la vez histórico y activo en el presente ya que la práctica se materializa por un conjunto de disposiciones aprendidas e interiorizadas que permiten tanto la reproducción de las estructuras sociales, como explicar sus cambios a través de los fenómenos de agencia. La Teoría de la Práctica de Bourdieu nos permite ver los procesos tecnológicos en su contexto social, cómo productos y productores de habitus en una cultura vista cómo un proceso histórico en un mundo de relaciones sociales y económicas contingentes. La relación cotidiana entre objetos, agentes y estructuras que se establece a través del habitus nos permite conocer las fuerzas sociales y las relaciones que condicionan la cultura material (Dietler y Herbich 1998). La estructura y la agencia son mediatizadas por la praxis diaria, y en el caso del proceso tecnológico, por la práctica cotidiana de fabricación, uso, intercambio y abandono de los objetos. La tecnología así concebida se convierte en un fenómeno cultural complejo, inserto en visiones de un mundo históricamente contingente, en acciones sociales estratégicas y en fenómenos de agencia. Los procesos tecnológicos superan el análisis del mero soporte físico para conectarse íntimamente con los fenómenos sociales.

En definitiva, desde esta perspectiva, el proceso tecnológico se conceptualiza del modo siguiente: a) El engranaje de relación de los actores sociales con sus condiciones materiales de existencia mediante la práctica diaria; b) el concepto bourdiano de habitus posibilita el análisis de su interacción y engranaje con la producción y reproducción social. Comprende tanto las técnicas concretas de fabricación o uso de un objeto, como la confección de metáforas sobre la interacción social; c) la tecnología engloba las prácticas cotidianas sobre la realidad material, fruto del conjunto de relaciones sociales significadas y negociadas que, a su vez, estructuran dichas prácticas; d) al presentar cierto comportamiento fractal un análisis a escala micro del proceso tecnológico nos aproxima al papel del agente y de los grupos $\mathrm{y}$ con ello a las interacciones generadas en el espacio social donde se ubican las actividades diarias. El análisis a escala micro permite una vinculación con las transformaciones sociales a escala macro (Chase 1989; Johnson 1989; Dobres y Hoffman 1994; Dobres 1995), porque posibilita la compresión del proceso de agencia y su relación con las estructuras, incluso en el ámbito de la cultura material arqueológica; e) la aproximación al proceso tecnológico debe ser contextual. Es fundamental analizar los aspectos sociales, políticos, simbólicos, ambientales y económicos en el contexto de las relaciones sociales, las estructuras de poder, los esquemas de racionalidad, así como el funcionamiento de los procesos en la producción y reproducción de los esquemas sociales (Pfaffenberg 1988; Hodder 1994; Dobres y Hoffman 1999; Dobres 2000).

Esta línea de trabajo nos permite reubicar conceptos esenciales para el análisis tecnológico como el de secuencias o cadenas operativas y su correlación social y el de procesos de aprendizaje, transmisión de conocimientos y savoir faire. Desarrollada en gran parte por autores que podrían incluirse dentro de la Escuela de la Antropología de las Técnicas (Balfet 1991; Lemonnier 1992, 2004; Gosselain 1992, 2010; Mahias 1993; Creswell 2010), nos acerca a los esquemas conscientes e inconscientes del proceso tecnológico, al considerar tanto la caracterización físico-química de los objetos, procesos y herramientas como su condición de representación simbólica de otros conceptos intelectuales existentes en la sociedad. Implica un nuevo enfoque tanto en el análisis del propio objeto como en el de la relación existente entre los comportamientos técnicos y sociales. El objeto se considera una construcción eminentemente social y no la simple trasformación de una materia presente en la naturaleza. El enfoque conduce, a su vez, al análisis de fenómenos de estabilidad, reproducción e innovación tecnológica. Promueve el análisis tanto de los aspectos físicos y más obvios de la cultura material, como de los elementos más sociales en relación con su fabricación, uso, intercambio y

Trab. prehist., 71, N. ${ }^{\circ}$ 1, enero-junio 2014, pp. 7-22, ISSN: 0082-5638

doi: $10.3989 /$ tp. 2014.12121 
abandono. La tecnología es concebida como un sistema donde realidad social y materia natural se transforman.

Se insiste en que el proceso tecnológico no es una mera secuenciación de acciones físicas, sino la incorporación de complejos esquemas mentales aprendidos a través de la tradición. Ello concierne a los materiales con qué se fabrican los objetos, a la manera cómo se producen y usan (incluyendo el conjunto de acciones técnicas, los espacios, los tiempos, etc.), a las personas y a todo el proceso de aprendizaje (Lemonnier 1993). Se compatibiliza con el énfasis en un análisis exhaustivo de las acciones tecnológicas físicas en relación al mundo material, en cuanto estrategia global de estudio de la dimensión material y social del fenómeno. Las técnicas, materiales y formas están organizadas en códigos construidos socialmente por lo que las opciones tecnológicas observadas alcanzan su verdadero sentido en el espacio social donde están insertas. Muchas de las decisiones técnicas no dependen tan claramente de criterios estrictamente funcionales como de los esquemas mentales (symbolic system) o los sistemas socialmente estructurados de organización, secuenciación, definición y transmisión de las acciones técnicas (Pelegrin 1988; Gosselain 1992; Lemonnier 1993).

Siguiendo en parte a Lemonnier $(1989,1992)$ el proceso tecnológico, y cualquier acción técnica, combina componentes materiales y de significado social. Cuatro se incluyen en el análisis de la trasformación mecánica o proceso técnico y un quinto se relaciona con el conocimiento tecnológico: 1) Las características físico-químicas de la materia o materias primas del objeto, que influyen en el proceso tecnológico, sin olvidar que, siendo percibidas y usadas en el seno de una sociedad, no alcanzan todo su significado sin considerar su constitución social. Es decir que es imprescindible analizar los conocimientos de cada sociedad sobre su entorno y sus recursos y el modo como los interioriza; 2) la energía o fuerzas necesarias para transformar la materia, también socialmente constituida; 3) los objetos realizados y las herramientas utilizadas; 4) los gestos técnicos que se organizan secuencialmente y se analizan mediante las herramientas analíticas derivadas del concepto de cadena operativa, analizando en profundidad los niveles que permiten jerarquizar, estructurar y organizar tanto la secuencia de gestos técnicos como los mismos ges- tos; 5) los conocimientos técnicos necesarios en los procesos de configuración y uso de los objetos son dobles. Consisten en las representaciones mentales de las formas artefactuales a desarrollar y de la materia a transformar y en el registro mental de las modalidades de acción para la consecución técnica. Los agentes que ejecutan las acciones tecnológicas pueden tener conocimientos, a nivel consciente o inconsciente, propios del individuo o del grupo. No son estáticos, sino que van evolucionando a partir de las innovaciones de los agentes, fruto de su experiencia y savoir faire, así como de influencias externas que incorporan nuevos materiales, técnicas, motivaciones socio-económicas o ideológico-simbólicas. A su vez, en los conocimientos técnicos cabría diferenciar el savoir faire del aprendizaje. El primero, también denominado conocimiento operativo y secuencial, resulta de las representaciones mentales y las acciones realizadas sobre la materia. El aprendizaje incluye todos los aspectos que suponen la transmisión y adopción del conocimiento y los saberes técnicos: espacios de aprendizaje, tiempo, forma de aprendizaje, espacio social de actuación, agentes, etc. (Pelegrin 1985).

Lemonnier (1983) conceptualizó estos cinco aspectos como Sistemas Tecnológicos, considerando tres niveles: 1) La interacción de todos los elementos a escala interna, en una lógica de la secuencia de acciones y dinámicas técnicas. El constante reajuste de esas múltiples interacciones requiere un análisis conjunto para ser relevante; 2) un análisis de las interrelaciones con el resto de procesos tecnológicos que se generan en una sociedad. El trasvase de conocimientos, soluciones técnicas y materiales de unos procesos tecnológicos a otros es más común de lo que en inicio podría parecer. De ahí que el análisis completo de un proceso tecnológico requiera compararlos todos y, en especial, estudiar su compatibilidad y coherencia. Ello permite incidir en el carácter fractal de la tecnología; 3) la conceptualización de las relaciones como parte de la acción social $\mathrm{y}$, por tanto, imbricadas con otras variables sociales, económicas, ideológicas, etc. Estudiar las relaciones entre la cultura material y la sociedad supone analizar las condiciones de coexistencia y transformaciones recíprocas entre el proceso técnico y la sociedad en la que está inmerso.

Los conceptos de elección tecnológica y representation technologique completan nuestra exposición teórica. El primero se ha desarrollado 
frecuentemente a partir de las nociones de efectividad, funcionalidad o condicionantes naturales (Franken 1971; Van der Leeuw 1976; Rye 1981; Franken y Kalsbeek 1984; Arnold 1985; Rice 1987; etc.). También se ha establecido que las acciones tecnológicas están conformadas por elecciones significativamente configuradas y relacionadas con las estructuras mentales de los grupos (Levi-Strauss 1986). En paralelo a ambas tradiciones, un amplio abanico de análisis han ido incorporando variables sociales, identitarias y simbólicas y no exclusivamente tecnológico-funcionales o condicionadas por las propiedades fisicoquímicas de los materiales.

El proceso de elección exige contar con alternativas (Van der Leeuw 1993) sin descartar que el mantenimiento de los procesos tecnológicos tal y como fueron aprendidos sea una de ellas. El punto de partida de un proceso de selección es, pues, el análisis de las alternativas que deben valorarse en un contexto social determinado que condiciona y delimita cualquier elección (Colomer 1995). Las múltiples opciones viables en teoría, se reducen en la práctica al pequeño grupo considerado por los agentes, muy condicionados por sus contextos de aprendizaje, tradición y espacio social. Sin embargo, no creemos que las secuencias productivas sean rígidas, predictivas y determinadas por la tradición, el espacio social y los contextos de aprendizaje. En cada fase del proceso productivo, se generan variantes, elecciones nuevas y decisiones diferentes a las tradicionales. La elección puede variar en cada fase de la cadena operativa y depender de múltiples factores. Irían desde las condiciones materiales hasta variaciones en los productos finales, fruto de cambios de uso o de costumbres, pasando por variaciones tecnológicas a partir de la experimentación individual tras la etapa de aprendizaje que pueden integrarse en el savoir faire y en el esquema psicomotor colectivo (Gosselain 1992).

Esa base permite diseñar estrategias que combinen las variables fisicoquímicas, tecnológicas, sociales, simbólicas, perceptivas, identitarias, etc, en una relación de doble dirección estructurada y estructurante para abordar cuestiones como ¿dónde y en qué tipo de técnicas se observan dichos procesos de elección? ¿Son reales o fruto de la interpretación del investigador? ¿Cuáles son las opciones disponibles? ¿Qué relación hay entre su adopción o rechazo y la coherencia social y cultural?¿Qué consecuencias tecnológicas, eco- nómicas, sociales y simbólicas se derivan de dichas elecciones? ¿Cómo se produce el proceso de elección? ¿Cómo se percibe dicha elección? ¿En qué se basa?, ¿Cómo se resuelve la dicotomía elección y tradición o innovación y resistencia? etc. La elección está intrínsecamente ligada al medio social donde se desarrolla el sistema tecnológico. Como siempre se guía por criterios coherentes socialmente, por lo que no es imprescindible que se relacionen con una gran eficacia tecnológica pero no por ello es adecuado hablar de elecciones tecnológicas arbitrarias. Todas las elecciones son coherentes con el espacio social que ocupan.

Cualquier elección, al derivar de una acción social, puede incorporar signos o símbolos que únicamente tienen sentido en el contexto social donde se fabrican, usan, intercambian o abandonan los objetos. Motivos o técnicas decorativas, determinadas formas o uso de materiales, etc., pueden trascender los aspectos funcionales para adentrarse, como planteó Hodder (1982), en el ámbito socio-ideológico-simbólico de una comunidad. Sin embargo, dichos símbolos no tienen por qué reducirse a los aspectos formales o estilísticos de los objetos, sino que también pueden intervenir en el proceso de producción, uso, intercambio y abandono, en una relación de doble dirección entre el mundo material y el ideológico simbólico (Gosselain 1992) (2). Ello explica, en muchos casos, que algunas decisiones técnicas no sean ni las más operativas, ni las más productivas desde un punto de vista funcional y que, desde el punto de vista de la efectividad, puedan considerarse arbitrarias o irracionales. Un análisis estrictamente utilitarista conduciría a un error de interpretación sino se analiza la vertiente social de dicha elección. En ese nivel alcanza su coherencia y estructuración, incorporando las interrelaciones entre agentes, creencias, ideas, relaciones sociales, etc. Ahí radica la importancia del estudio de las elecciones técnicas como fenómenos socialmente estructurados, mucho más allá de la intrínseca lógica funcional o de las características fisicoquímicas de los materiales.

La consideración de las relaciones sociales no debe llevarnos a un análisis unidireccional de la acción social versus la decisión técnica. Lo que

(2) La expresión savoir faire se prefiere a la de knowledge por relacionarse mejor con las fuentes intelectuales de las que parte el discurso. 
se defiende es que, una elección tecnológica determinada, las características físico-químicas de los materiales, la función y el uso diario exigido al objeto no son elementos neutros, sino de carácter estructurante. De ahí la importancia de conjugar estrategias que vayan, desde el análisis profundo de los materiales, las acciones técnicas y su secuencia, hasta la vertiente social tras cada variable elegida.

Finalmente, nos gustaría incluir el concepto de representation technologique nos interesa en sus tres variantes (Lemonnier 1992): 1) Las operaciones mentales, muchas veces inconscientes, que hay en toda acción tecnológica. Sustentan los movimientos, posiciones de las manos, etc., es decir, cada acción de los agentes en el proceso tecnológico. Estas operaciones inconscientes, generadas a partir del habitus tecnológico, tienen un papel fundamental en la transmisión del conocimiento. Esta suele realizarse por un método repetitivo, no normativo, donde los esquemas mentales inconscientes y sus derivaciones psicomotrices se van introduciendo poco a poco, a través de la práctica diaria, en el aprendiz mediante la imitación del maestro; 2) los modelos mentales de la secuencia y ordenación de la acción o acciones tecnológicas, incluyendo los materiales, las herramientas, el espacio destinado a la operación, la posición del agente, la ordenación y la propia secuencia. Muchos de estos modelos no son exclusivos de una acción tecnológica concreta, sino que dependen de factores relacionados con otros procesos tecnológicos o con otros esquemas de distribución y organización de los trabajos u otras estructuras sociales; 3) los contenidos o informaciones de tipo ideológico, social o simbólico que contienen las acciones tecnológicas. Dichas informaciones funcionan en un entramado supratecnológico de significados que afecta a la totalidad de los esquemas y modelos de significación y simbología de la comunidad que se analiza.

La idea del funcionamiento transversal de los esquemas de significación y de los procesos tecnológicos en el seno de una comunidad nos lleva a un planteamiento fractal de la tecnología, de su imbricación social y de los símbolos o significados que se estructuran con ella. Esto demanda un planteamiento global del fenómeno tecnológico y abre la posibilidad de desarrollar análisis de escala (Marquart 1992) que permitirían completar el estudio del proceso tecnológico y su comportamiento fractal. En este sentido, la dinámica so- cial que opera a escala micro conduce a los procesos a escala macro (Chase 1989; Dobres y Hoffman 1994). La interacción de escalas opera en los ejes vertical y horizontal, pues afecta a cada actividad tecnológica y social de un mismo grupo.

Muchos de los elementos incluidos en estos tres niveles del concepto de representation se estructuran de manera inconsciente en diferentes estadios por lo que varía su grado de permeabilidad a los cambios. En ocasiones, actúan como elementos de permanencia o estabilidad tecnológica y por ende social y cultural. Ciertas acciones tecnológicas son menos susceptibles a los cambios que otras que no han sido tan marcadas desde el aprendizaje y, sobre todo, que no se han incorporado con tanta insistencia en los esquemas psicomotrices técnicos requeridos para ejecutarla.

El concepto de habitus bourdiano adquiere una especial relevancia en ese aspecto inconsciente del conocimiento, claramente enfatizado por Lemonnier, en tanto herramienta eficaz para el análisis de la elección tecnológica y sus posibles derivaciones sociales.

\section{RECAPITULACIÓN Y HERRAMIENTAS INTERPRETATIVAS}

Nuestro énfasis en el carácter eminentemente social de la tecnología no implica que excluyamos de su interpretación los condicionamientos medioambientales, la función de los productos, la relación coste-beneficio o las exigencias técnicas del propio desarrollo tecnológico.

Entendemos que deben analizarse desde la perspectiva de su integración en un contexto social históricamente contingente, donde se da el grueso de las interrelaciones tecnológicas. Lo que confiere sobre todo esa gran capacidad estructurante y estructurada al proceso tecnológico es la rutina cotidiana de fabricación, uso, intercambio $\mathrm{y}$ abandono de objetos en un espacio social, repetida por las personas desde la infancia hasta la madurez. De ahí su gran potencial como herramienta interpretativa de las comunidades pretéritas.

Una premisa central de este desarrollo teórico es que las personas dan un cierto significado a su mundo y en parte, lo transforman a partir de las experiencias socialmente constituidas generadas en el trabajo diario sobre un determinado material 
para fabricar y usar un objeto (Dobres 2000, 2010). La aproximación de los seres humanos a la percepción y estructuración de su mundo sería tan materialista como idealista (Conkey 1993). A través del estudio de los objetos arqueológicos, entendidos desde esta perspectiva, podremos adentrarnos en la compleja práctica social que supone el proceso tecnológico, así como en todas sus implicaciones en relación a los esquemas de racionalidad, a las praxis sociales, a las relaciones de poder, a las bases económicas, etc.

Concebimos la tecnología como una construcción social donde la acción tecnológica está enraizada en un universo de comportamientos y significados socialmente establecidos. Mantenemos, pues, una base epistemológica materialista, alejada de las estrategias exclusivamente hermenéuticas (Shanks y Tilley 1987). A partir de la materialidad, ya sea como gesto u objeto, se pueden analizar los esquemas mentales aprendidos a través de la tradición y que abarcan conceptos tan globales como los de uso, fabricación o significado.

Proponemos la integración del enfoque analítico sobre el objeto, su fabricación y uso con una postura teórica que profundice en la complejidad de las relaciones bidireccionales entre sociedad y tecnología, entre objetos y personas, entre condicionantes materiales y estructuración de los esquemas de racionalidad y entre estructura y agencia. Dichos aspectos interpretativos no se deducen directamente de la materialidad del registro arqueológico, sino que requieren un engranaje conceptual bien definido, alcanzable, entre otras estrategias, a partir de tres líneas de desarrollo: un análisis contextual, un nuevo concepto de cadena operativa y la inclusión de conceptos específicos de savoir faire, aprendizaje y trasmisión de conocimientos.

\subsection{Estrategias de análisis contextual}

Hemos optado por una estrategia en red para incorporar los diferentes conceptos a analizar y lograr una rápida conexión entre materialidad, estructuras, agentes y relaciones de agencia. Como ejemplo de un modelo de red de una fase tecnológica, mostramos un análisis de la producción cerámica (Fig. 1). Se valoran los aspectos materiales e inmateriales, ya que ambos se generan simultáneamente y se estructuran en conjun- to. La continua retroalimentación asumida entre materialidad e inmaterialidad obliga a desarrollar enfoques teóricos, metodológicos e instrumentales para analizar el papel activo de ambas realidades e incorporar las conexiones y relaciones que se generan. Por ello, junto a la localización de los restos cerámicos o los tiempos de ejecución, valoramos la identidad, el habitus, los condicionantes de género o aprendizaje, cuyo análisis intrínseco ya requiere una consideración previa de aspectos materiales e inmateriales.

El análisis de la materialidad (campos ubicados en la parte superior del esquema) y la interacción estructurante y estructurada entre la producción cerámica y otras esferas del espacio social (campos ubicados en la parte inferior del esquema) van generando las redes y relaciones multidireccionales que permiten analizar la complejidad de elementos que juegan un papel activo en la producción cerámica y en las personas que la llevan a cabo. Más allá del marco general propuesto el carácter históricamente contingente de la concreción de los campos y de las relaciones generadas en la red exige el análisis concreto del proceso tecnológico para acabar dibujando la complejidad o variabilidad de la red.

Analizamos ese conjunto de interacciones a través del "proceso tecnológico" o "estrategia productiva". Incluye a) el conjunto de acciones físicas, aprendidas socialmente, que se dan en la secuencia de transformación de la materia seleccionada a la consecución del objeto; b) aspectos relacionados con los espacios y tiempos sociales donde se realizan estas acciones; c) los agentes que intervienen, los procesos de aprendizaje y habitus adquiridos; d) las estructuras sociales, económicas e ideológicas en las que se insertan y e) el conjunto de interacciones estructuradas y estructurantes entre cada uno de estos elementos.

Lo esencial de la propuesta es la reubicación de los objetos y las personas en el centro del planteamiento del proceso productivo. Es decir, la relación generada entre la materialidad y las personas que interactúan con ella, la perciben e interpretan. Las personas son centrales porque fabrican, usan, intercambian y abandonan los objetos. Perciben y adquieren experiencias y conocimientos a través de esas actividades. Su quehacer cotidiano va estructurando y modelando el mundo. Interrelacionan las condiciones materiales de fabricación y uso cotidiano de los objetos con valores, esquemas de racionalidad y significados. 


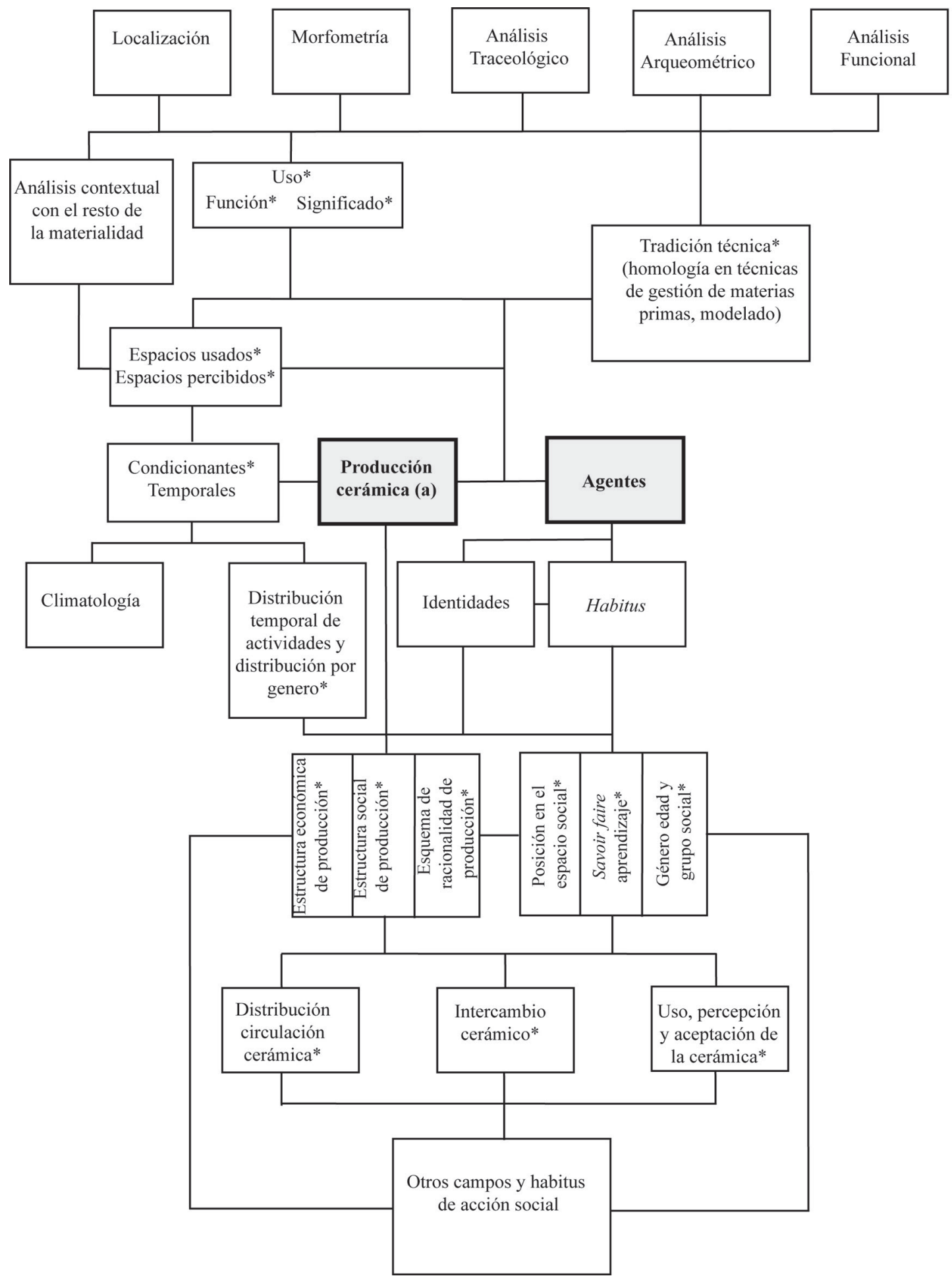

a) Producción cerámica: obtención y gestión de la materia prima, proceso de modelado y proceso de cocción de la cerámica. Interrelación con las experiencias y conocimientos que se generan en cada uno de los procesos.

* Elementos relacionados contextualmente, estructurados y estructurantes y con contingencia geográfica y temporal

Fig. 1. Red conceptual del Proceso tecnológico productivo de la cerámica a mano. 
Con ello, la atención regresa a las relaciones dinámicas en los procesos de transformación material y social y, por ende, de cambio o reproducción, pues las experiencias vitales de las personas, como individuos o colectivo, van cambiando.

Además los objetos pueden funcionar, como las personas, como auténticos agentes estructurados y estructurantes (Latour 2008). A través de ellos, creamos, modificamos y concebimos una determinada visión del mundo. Por ello, la cultura material debe ser considerada como parte activa en la reproducción, mantenimiento o subversión de una sociedad.

El análisis de agentes y objetos abre la puerta, a través de los conceptos de habitus Bourdiano o de rutinas socialmente aprendidas (estructuradas y normativizadas), a los esquemas de racionalidad, los valores, las relaciones de poder, el espacio social que ocupa la actividad y los agentes, así como a los cambios y resistencias generados.

\subsection{Un nuevo concepto de cadena operativa}

Definimos la cadena operativa como el conjunto de acciones técnicas y operaciones físicas aprendidas socialmente que se dan en la secuencia de transformación, fabricación, uso y reparación de un objeto, cultural y socialmente estructurado a partir de unos recursos naturales también socialmente concebidos. Así entendida se convierte en la herramienta idónea para el análisis tecnológico ya que correlaciona materia, pensamiento y organización social.

Este concepto de cadena operativa incluye la secuencia de gestos y acciones técnicas para fabricar y usar un objeto y, a través de ella, incorpora el bagaje de conocimiento tecnológico necesario, las alternativas técnicas posibles, el propio savoir faire del agente o del colectivo, las decisiones, juicios técnicos y elecciones, así como las rutinas diarias de aprendizaje, práctica y uso, concebidas desde perspectivas de habitus bourdiano.

La primera fase de análisis interpreta la secuencia de operaciones físicas de transformación de recursos naturales en objetos y su posterior uso. La interpretación arqueológica partiría, básicamente, de las trazas materialmente observables de dichas acciones en los objetos y de los cambios físico-químicos producidos durante su pro- ceso de fabricación y uso. Esta secuencia de operaciones es fruto de un proceso rutinario de aprendizaje, de la elección de unas opciones técnicas de fabricación y de unos usos socialmente estructurados, así como del espacio social donde se encuadra. Por ello, las acciones técnicas no reflejan únicamente la trasformación física de la materia. Interactúan mediante el aprendizaje, la rutina diaria, el habitus y el espacio social que ocupan con las estructuras económico-sociales e ideológicas del individuo, del grupo y de la comunidad donde se inserta el proceso tecnológico. Dicha interacción, realizada a través del agente, de sus actos y del objeto, está condicionada por aspectos materiales y culturales.

El análisis y la comparación de cadenas operativas permiten analizar los procesos técnicos y acciones técnicas y los siguientes aspectos: a) La heterogeneidad, homogeneidad y homología en cada fase de las cadenas operativas que, como se verá, permitirán definir variaciones estructurales o secundarias en las cadenas operativas; b) una aproximación al aprendizaje y a la transmisión de conocimiento a través de variantes como la psicomotricidad interiorizada y las variaciones estructurales o secundarias; c) la elección tecnológica, bien mediante préstamos tecnológicos o fenómenos de agencia d) el concepto de savoir faire y tradición tecnológica y e) el carácter transversal y fractal de las dinámicas tecnológicas del grupo.

\subsection{La inclusión de los conceptos de savoir faire, aprendizaje y trasmisión de conocimientos}

Como se sabe, una premisa central en nuestro planteamiento es que las personas dan un determinado significado a su mundo $\mathrm{y}$, en parte, lo transforman a partir de experiencias socialmente constituidas, generadas cuando, en su quehacer cotidiano, trabajan un determinado material con el fin de fabricar y usar un objeto. No concebimos una técnica como un mero gesto, sino siempre como una representación física de elecciones y esquemas mentales aprendidos a través de una tradición tecnológica inserta, a su vez, en un contexto social determinado (Lemonnier 1992). Sin embargo, esta tradición tecnológica no es ni mucho menos estable sino que participa de un constante diálogo con fenómenos de cambio y

Trab. prehist., 71, N. ${ }^{\circ}$ 1, enero-junio 2014, pp. 7-22, ISSN: 0082-5638

doi: $10.3989 /$ tp. 2014.12121 
estabilidad, o incluso de resistencia tecnológica. Ello nos obliga al análisis del savoir faire tecnológico y de su mantenimiento o modificación durante los procesos de trasmisión del conocimiento, actuantes en cada momento en la comunidad.

El modelado a mano de la cerámica, seleccionado como caso de estudio, ocurre en contextos domésticos donde las mujeres son las que protagonizan la mayor parte de los estadios de la secuencia operativa de producción. En estos contextos, el conocimiento requerido suele trasmitirse de generación en generación a través de un aprendizaje en el grupo familiar. Desde niña, la alfarera aprende el savoir faire trasferido por la madre, la hermana o una parienta cercana, que a la vez aprendieron de sus madres, y así sucesivamente durante generaciones. El aprendizaje se combina internamente con otros tecnológicos (conocimiento de las prácticas culinarias, agricultura, recolección, etc.) y sociales, así como con los valores éticos y la cosmovisión del grupo. En todos ellos, los fenómenos de habitus actuarían significativamente a la hora de fijar prácticas, conocimientos y valores.

En general, la cercana interacción entre maestra y pupila durante el aprendizaje permite la transmisión de los esquemas físico-motores necesarios para adquirir el savoir faire relacionado con el modelado de la cerámica. Esta fase dura hasta que las operaciones manuales, fruto de la práctica cotidiana, se vuelven casi automáticas, $\mathrm{y}$ el cerebro del aprendiz adquiere y memoriza un programa psicomotriz preciso.

El nivel social del proceso de aprendizaje deriva de que el savoir faire de la maestra engloba el conocimiento tecnológico del grupo. Este no es meramente individual sino de carácter social.

En este contexto de transmisión de conocimientos y valores, la innovación gestual no puede aparecer durante el aprendizaje, pues la maestra corrige de inmediato cada gesto no adecuado al patrón propuesto. Los gestos, normalizados a través de la práctica repetida de la actividad, llegan a ser gradualmente incorporados como un esquema psicomotor inconsciente. La innovación o la adopción de otras técnicas es virtualmente imposible, ya que requeriría un proceso de "desaprender" compensado con otro de "volver a aprender", algo difícil de imaginar sin un factor causante de medidas tan drásticas (Gosselain 2002). La fuerte interiorización de algunos gestos motores durante el proceso de aprendizaje expresa intensas dinámicas de habitus, generadoras de un proceso de modelado muy estable con una baja permeabilidad a cambios, préstamos, modas e innovaciones. El profundo enraizamiento de ciertos procesos en las tradiciones tecnológicas, les confiere una enorme estabilidad y resistencia al cambio y a la permeabilidad de nuevas ideas técnicas.

La interpretación y el significado de las variables tecnológicas se estructuran identificando las continuidades y rupturas que se dan en los patrones tecnológicos. Dichas variables pueden considerarse individuales o debidas a cambios en una tradición tecnológica. Para analizar estas últimas resulta sumamente eficaz identificar las cadenas operativas a partir de la heterogeneidad entre ellas, de su homogeneidad o incluso de su homología.

Las variaciones en las cadenas operativas de fabricación, según su alcance, son estructurales o parciales. Las variaciones estructurales o estratégicas en el proceso tecnológico no pueden suprimirse, cancelarse o remplazarse sin modificar de modo significativo el proceso o el resultado final (Lemonnier 1992). Se corresponden con cambios, cuyas modificaciones profundas en las secuencias de fabricación, condicionan a su vez la introducción de nueva infraestructura tecnológica y alteran el savoir faire aprendido. Por ello, deben correlacionarse con trasformaciones más amplias en el seno del grupo. Las variaciones parciales o secundarias no cambian de manera significativa los procesos de aprendizaje, ni modifican las técnicas, ni exigen adoptar una nueva infraestructura tecnológica.

La documentación de unas variaciones u otras supondrá cambios interpretativos substanciales. La identificación de variaciones de tipo estratégico o estructural reflejará cambios profundos en la tradición tecnológica relativos al proceso de modelado, a las infraestructuras $\mathrm{y}$, especialmente, a los esquemas psicomotrices. Es decir, afectará a las estrategias de aprendizaje, al savoir faire del grupo $\mathrm{y}$, en consecuencia, puede implicar modificaciones substanciales en algunos elementos del espacio social donde se fabrican los objetos. En cambio, las variaciones de tipo secundario remitirán a modas, variaciones estéticas, gustos $\mathrm{o}$ elecciones más individuales sin implicar necesariamente profundas modificaciones del savoir faire del grupo, ni de los procesos de aprendizaje 
ni, por consiguiente, del espacio social donde se enmarcan.

Explicar las variaciones en las secuencias operacionales equivale a explorar el espacio social en el que se insertan. Ello nos permite identificar los vínculos pertinentes entre fenómenos técnicos y factores de orden social, pues la elección de una acción técnica siempre es lógica en el marco social donde se desarrolla, aunque su eficacia tecnológica parezca mejorable desde fuera del grupo. La estrategia correcta no es considerar la presencia-ausencia de algún rasgo técnico, sino entender el significado de rupturas tecnológicas, que pueden variar de una comunidad a otra o de un periodo a otro. En lugar de interpretaciones mecanicistas, es obligado el análisis contextual de todo el proceso cerámico y de su espacio social.

En suma el objetivo de la línea discursiva desarrollada en este trabajo ha sido subrayar como el análisis tecnológico supone ir más allá de identificar los sistemas de fabricación. A partir de la reconstrucción de la tecnología podemos aproximarnos al espacio social y adentrarnos, a través de los conceptos de hábitus, práctica diaria, estrategias de aprendizaje, etc., en fenómenos relacionados con los contactos intergrupales, con cuestiones de género, de identidad, con procesos de resistencia e innovación. Es decir, podemos conocer las dinámicas sociales de los grupos humanos. El bagaje teórico del artículo ha pretendido dar cobertura al deseo de ir más allá de la mera materialidad pero sin obviar el análisis de esa materialidad que nos permite profundizar en nuestras interpretaciones sociales.

\section{AGRADECIMIENTOS}

El artículo es parte de la transferencia de conocimientos del proyecto de investigación "Vivir entre islas: paisajes insulares, conectividad y cultura material en las comunidades de las Islas Baleares durante la Prehistoria Reciente (2500123 BC)" (HAR 2012 32602) financiado por el Ministerio de Economía y Competitividad.

\section{BIBLIOGRAFÍA}

Arnold, D. E. 1985: Ceramics theory and cultural process. Cambridge University Press. Cambridge.
Balfet, H. 1991: Observer l'action technique. Des chaines operatoires, pour quoi fare? Centre National de la Recherche Scientifique. Paris.

Bettinger, R. 1980: "Explanatory/predictive models of hunter-gatherer adaptation". Advances in Archaeological Method and Theory 3: 189-225.

Binford, L. 1962: "Archaeology as Anthropology". American Antiquity 28: 217-225.

Binford, L. 1964: "A consideration of archaeological research design". American Antiquity 29 (4): 425440.

Binford, L. 1965: "Archaeological systematics and the study of culture process". American Antiquity 31: 203-210.

Bourdieu, P. 1988: La distinción. Criterio y bases sociales del gusto. Taurus. Madrid.

Bourdieu, P. 1991: El sentido práctico. Taurus. Madrid.

Bourdieu, P. 1997: Razones prácticas sobre la teoría de la acción. Anagrama. Barcelona.

Chase, A. K. 1989: "Domestication and domiculture in northern Australia: A social perspective". En D. R. Harris, y G. C. Hillman (eds.): Foraging and Farming: The Evolution of Plant Domestication. Unwin Hyman. Londres: 42-54.

Childe, V. G. 1936: Man Makes Himself. Collins. London.

Childe, V. G. 1956: Society and Knowledge. Harper Brothers. New York.

Childe, V. G. 1988: Los orígenes de la civilización. Fondo de Cultura Económica. Madrid.

Clarke, D. L. 1976: “Archaeology. The loss of inocence". Antiquity 47: 6-18.

Clarke, D. L. 1984: Arqueología analítica. Bellaterra. Barcelona.

Colomer, E. 1995: Pràctiques socials de manufactura ceràmica: análisis morfométriques i tecnològiques al sud-est de la Peninsula Ibérica, 2200-1500 cal. ane. Tesis doctoral Microforma, Départament de Prehistoria, Universitat Autonoma de Barcelona. Barcelona.

Conkey, M. 1993: "Humans as materialists and symbolists: Image-making in the upper paleolithic". En D. T. Rasmunsen (ed.): The Origin and Evolution of Humans and Humanness. Jones and Barlett. Boston: $95-118$.

Cresswell, R. 2010: "Techniques et culture: les bases d'un programme de travail". Techniques et Culture 54-55: 20-45

Daniel, G. 1963: The ideas of Prehistory. The World publishing Company. Cleveland.

Daniel, G. 1967: The Origins and Growth of Archaeology. Penguin. Harmondsworth.

Daniel, G. 1987: Un siglo y medio de arqueología. Fondo de Cultura Económica. México.

Dietler, M. y Herbich, I. 1998: "Habitus, techniques, style: an integrated approach to the social unders- 
tanding of material culture and boundaries". En M. Stark (ed): The Archaeology of Social Boundaries. Smithsonian. Washington: 232-279.

Dobres, M. A. 1995: "Gender and prehistoric technology: on the social agency of technical strategies". World Archaeology 27: 25-49.

Dobres, M. A. 2000: Technology and social agency. Blackwell. Londres.

Dobres, M. A. 2010: "Archaeologies of technology". Cambridge Journal of Economics 34: 103-114.

Dobres, M. y Hoffman, C. 1994: "Social agency and the dynamics of prehistoric technology". Journal of Archaeological Method and Theory 1 (3): 211-258.

Dobres, M. y Hoffman, C. 1999 The social Dynamics of Technology. Practice, Politics and World Views. Smithsonian Institution Press, Washintong.

Franken, H. J. 1971: "Analysis of methods of potmaking in archaeology". Harvard Theological Review 64: 227-255.

Franken, H. J. y Kalsbeek, J. 1984: "Iron age pottery from Harem (Nothern Brabant, The Netherlands)". Newsletter of the Departament of Pottery Technology (Leiden Universty) II: 17-26.

Fritz, J. M. y Plog, F. T. 1970: "The nature of archaeological explanation". American Antiquity 35 (4): 405-412.

Giddens, A. 1979: Central Problems in Social Theory: Action, Structure, and Contradiction in Social Analysis. University of California Press. Berkeley.

Giddens, A. 1984: The Constitution of Society: Outline of the Theory of Structuration. Polity Press. Cambridge.

Gosselain, O. 1992: "Technology and Style: Potters and Pottery among Bafia Cameroon". Man 27 (3): 559-586.

Gosselain, O. 2002: Poteries du Cameroun meridional. Styles, techniques et rapports a l'identité. Centre National de la Recherche Scientifique. Paris.

Gosselain, O. 2010: "Exploring the dynamics of African pottery cultures". En R. Barndon, A. Engevik e I. Øye (eds.): The Archaeology of Regional Technologies. The Edwin Mellen Press. Lewiston.

Grau Mira, I. 2007: "Dinámica social, paisaje y teoría de la práctica. Propuestas sobre la evolución de la sociedad ibérica en el área central del oriente peninsular". Trabajos de Prehistoria 64 (2): 119-142.

Habermas, J. 1970: Toward a Relational Society. Student Protest, Science, and Politics. Beacon Press. Boston.

Hayden, B. 1995: "The Emergence of Prestige Technologies and Pottery". En W. K. Barnett y J. $\mathrm{W}$. Hoopes (eds.): The emergence of pottery production: Technology and Innovation in ancient societies. Smithsonian Institution Press. Washington: 257-265.
Hayden, B. 1998: "Practical and Prestige Technologies: The Evolution of Material Systems". Journal of Archaeological Method and Theory 5 (1): 1-55.

Hernando Gonzalo, A. 1992: "Enfoques teóricos en arqueología". SPAL 1: 11-37.

Hodder, I. 1982: Symbols in action. Cambridge University Press. Cambridge.

Hodder, I. 1989: The meanings of the things. Material Culture and Symbolic expression. One World Archaeology series 6, Harper Collins Academic.

Hodder, I. 1994: Interpretación en arqueología: Corrientes actuales. Crítica. Barcelona.

Ingold, T. 1988: "Comment to Testart". Current Anthropology 29 (1): 14-15

Ingold, T. 1990: "Society, nature and the concept of technology". Archaeological Review from Cambridge 9 (1): 5-17.

Johnson, M. 1989: "Conceptions of Agency in Archaeological Interpretation". Journal of Anthropological Archaeology 8: 189-211.

Latour, B. 2008: Reensamblar lo social Una introducción a la Teoría del Actor-red. Ed. Manantial. Buenos Aires.

Lemonnier, P. 1983: "La Description des Systèmes techniques. Une urgence en technologie culturelle". Techniques et Culture 1: 11-26.

Lemonnier, P. 1986: "The study of material culture today: Towards an anthropology of technical systems". Journal of Anthropological Research 5:147186.

Lemonnier, P. 1989: "Towards an anthropology of technology". Man 24: 526-527.

Lemonnier, P. 1992: Elements for an anthropology of technology. University of Michigan, Museum of Anthropology. Michigan.

Lemonnier, P. 1993: "Introduction to technological choices". En P. Lemonnier (ed.): Transformation in Material Cultures since the Neolithic. Routledge. London.

Lemonnier, P. 2004: "Mythiques chaînes opératoires". Techniques et culture 43-44: 25-44.

Leone, M. 1982: "Some opinions about recovering mind". American Antiquity 49: 742-760.

Leroi-Gourhan, A. 1964: La geste et la Parole. Technique et Langage. A. Michel. Paris.

Leroi-Gourhan, A. 1988: El hombre y la materia (Evolución y técnica I). Taurus. Madrid.

Leroi-Gourhan, A. 1989: El Medio y la Técnica (Evolución y Técnica II). Taurus. Madrid.

Levi-Strauss, C. 1986: La alfarera celosa. Paidós. Barcelona.

Mahias, M. 1993: "Pottery techniques in India. Technical variants and social choice". En P. Lemonnier (ed.): Technological choices. Transformation in material cultures since the Neolithic. Routledge. London: 157-180. 
Marcuse, H. 1968: Essays in Critical Theory. Beacon Press. Boston.

Marquart, W. 1992: “Dialectical Archaeology”. Journal of Archaeological Method and Theory 4: 101140.

Mauss, M. 1968: "Les techniques et la technologie". Euvres 3. Cohésion sociale et divisions de la Sociologie. Paris. Minuit: 250-256.

Mauss, M. 1979: Sociology and Psychology. Routledge and Kegan Paul. London.

Mitcham, C. 1994: Thinking through Technology: The Path between Engineering and Philosophy. University of Chicago Press. Chicago.

Olsen, B. 2003: "Material Culture after Text: ReMembering Things". Norwegian Archaeological Review 36 (2): 87-104.

Olsen, B. 2010: In Defense of Things: Archaeology and the Ontology of Objects. Altamira Press. Lanham.

Pelegrin, J. 1985: "Réflexions sur le comportement technique". En La signification culturelle des industries lithique. Actes du colloque (Liege 1984). Studia Praehistorica Belgica 4: 72-91.

Pelegrin, J. 1988: “A framework for analysing prehistoric stone tools manufacture and tentative application to some early lithic industries". En A. Berthelet y J. Chavaillon (eds.): The use of Tools in humans and non-humans primates. Clarendon Press. Oxford: 302-314.

Pfaffenberger, B. 1988: "Fetishized objects and humanized nature: towards an anthropology of technology". Man 23: 236-252.

Pippin, R. 1995: "On the Notion of Technology as Ideology”. En A. Feenberg y A. Hannay (eds.): Technology and The Politics of Knowledge. Indiana University Press. Bloominton: 43-61.

Rice, P. M. 1987: Pottery analysis: a sourcebook. University of Chicago Press. Chicago.

Ritzer, G. y Gindoff, P. 1994: "Agency-structure, micro-macro, individualism-holism-relationism: A metatheoretical explanation of theoretical convergence between the United States and Europe". En P. Sztompka (ed.): Agency and structure: Reorienting social theory. Gordon and Breach. Yverdon Switz: 3-23.

Roux, V. y Matarasso, P. 1999: "Craft and The Evolution of Complex Societies: New methodologies for modelling the organization of production, a Harappan example". En M. Dobres y C. Hoffman (eds.): The Social Dynamics of Technology, Practice, Politics and World Views. Smithsonian Institution Press. Washington: 46-70.
Rye, O. S. 1981: Pottery Technology: Principles and Reconstruction. Taraxacum. Washington D. C.

Sabloff, J. y Willey, G. 1967: "The Collapse of Maya Civilization in the Southern Lowlands: A Consideration of History and process". Southwestern Journal of Anthropology 23: 311-336.

Shanks, M. y Tilley, C. 1987: Reconstructing Archaeology. Cambridge University Press. Cambridge.

Testart, A. 1982: Les chasseurs-cueilleurs, ou l'origine des inégalités. Société d'Etnographie Nanterre Université Paris X. Paris.

Testart, A. 1988: "Some Major problems in the social anthropology of hunter-gatherers". Current Anthropology 29 (1): 1-32.

Thomas, D. H. 1974: “An Archaeological Perspective on Shosshonean Bands". American Anthropologist 76: 11-23.

Tilley, C. 1989: "Interpreting Material Culture". En I. Hodder (ed.): The meanings of the things. Material Culture and Symbolic expression. One World Archaeology series 6, Harper Collins Academic: 181-194.

Tilley, C. 1991a: Reading the material culture. Blackwell. Oxford.

Tilley, C. 1991b: Material Culture and Text. Routledge. London.

Torrence, R. 1983: "Time budgeting and huntergatherer technology". En G. Bailey (ed.): HuntherGatherer Economy in Prehistory: A European Perspective. Cambridge University Press. Cambridge: 11-22.

Trigger, B. 1989: "Hyperrelativism, Responsibility and the Social Sciences". Canadian Review of Sociology and Anthropology 26: 776-797.

Trigger, B. 1992: Historia del pensamiento arqueológico. Crítica. Barcelona.

Van der Leeuw, S. E. 1976: Studies in the technology of ancient pottery. Universiteit van Amsterdam. Amsterdam.

Van Der Leeuw, S. E. 1993: "Giving the potter a choice. Conceptual aspects of pottery techniques". En P. Lemonier (ed.): Technological choice. Transformations in material cultures since the neolitic. Routledge. London: 238-288.

Wallace, M. 1986: "Visiting the Past: History Museum in the United States". En S. Porter; S. Brier y R. Rosenzweig (eds.): Presenting the Past: Essays on the History and the Public. Temple University Press. Philadelphia: 137-161.

Watson, P. J.; Leblanc, S. A. y Redman, C. L. 1971: El método científico en arqueología. Alianza Editorial. Madrid. 